\title{
Asymptotic scaling laws for the irrotational motions bordering a turbulent region
}

\section{Article}

Accepted Version

Xavier, R. P., Teixeira, M. A. C. and da Silva, C. B. (2021)

Asymptotic scaling laws for the irrotational motions bordering a turbulent region. Journal Of Fluid Mechanics, 918. ISSN 00221120 doi: https://doi.org/10.1017/jfm.2021.296 Available at https://centaur.reading.ac.uk/97856/

It is advisable to refer to the publisher's version if you intend to cite from the work. See Guidance on citing.

To link to this article DOI: http://dx.doi.org/10.1017/jfm.2021.296

Publisher: Cambridge University Press

All outputs in CentAUR are protected by Intellectual Property Rights law, including copyright law. Copyright and IPR is retained by the creators or other copyright holders. Terms and conditions for use of this material are defined in the End User Agreement.

\section{www.reading.ac.uk/centaur}

\section{CentAUR}

Central Archive at the University of Reading

Reading's research outputs online 


\title{
Asymptotic scaling laws for the irrotational motions bordering a turbulent region
}

\author{
Ricardo P. Xavier ${ }^{1}$ and Miguel A. C. Teixeira ${ }^{2}$ and Carlos B. da \\ Silva $^{1} \dagger$ \\ ${ }^{1}$ LAETA, IDMEC, Instituto Superior Técnico, Universidade de Lisboa, Lisboa, Portugal \\ ${ }^{2}$ University of Reading, Department of Meteorology, Reading, United Kingdom
}

(Received xx; revised xx; accepted xx)

Turbulent flows are often bounded by regions of irrotational or non-turbulent flow, where the magnitude of the potential velocity fluctuations can be surprisingly high. This includes virtually all turbulent free shear flows and also turbulent boundary layers and is particularly true near the so-called turbulent/non-turbulent interface (TNTI) layer, which separates the regions of turbulent and non-turbulent fluid motion. In the present work we show that in the non-turbulent region, and for distances sufficiently far from the TNTI layer $x_{2}$, the asymptotic variation laws for the variance of the velocity fluctuations $\left\langle u_{i}^{2}\right\rangle(i=1,2,3)$, Taylor micro-scale $\lambda$, and viscous dissipation rate $\varepsilon$, depend on the shape of the kinetic energy spectrum in the infrared region $E(k) \sim k^{n}$. Specifically, by using rapid distortion theory (RDT) we show that for Saffman turbulence $\left(E(k) \sim k^{2}\right)$ we obtain the following asymptotic laws: $\left\langle u_{i}^{2}\right\rangle \sim x_{2}^{-3}(i=1,2,3), \lambda \sim x_{2}$ and $\varepsilon \sim x_{2}^{-5}$, while we confirm the classical results obtained by Phillips (1955) for Batchelor turbulence $\left(E(k) \sim k^{4}\right)$, with $\left\langle u_{i}^{2}\right\rangle \sim x_{2}^{-4}(i=1,2,3), \lambda \sim x_{2}$ and $\varepsilon \sim x_{2}^{-6}$. The new theoretical results are confirmed in direct numerical simulations (DNS) of shear-free turbulence, and are independent of the Reynolds number. Therefore these results are expected to be valid in other flow configurations such as in turbulent planar jets or wakes, provided the kinetic energy spectra in the turbulence region obeys a Batchelor or a Saffman spectrum.

Key words: Authors should not enter keywords on the manuscript, as these must be chosen by the author during the online submission process and will then be added during the typesetting process (see http://journals.cambridge.org/data/relatedlink/jfmkeywords.pdf for the full list)

\section{Introduction}

Turbulent flows are often bounded by regions of non-turbulent motion (Pope 2000). Examples include virtually all canonical turbulent free shear flows i.e. turbulent mixing layers, turbulent jets, and turbulent wakes, but the same is true of turbulent boundary layers. In these flows the non-turbulent (NT) and turbulent (T) flow regions are separated by the turbulent/non-turbulent interface (TNTI), which is a very sharp and highly contorted layer (Bisset et al. 2002; Westerweel et al. 2009; da Silva et al. 2014). Its sharpness is connected with an abrupt transition in the vorticity field observed between $\mathrm{T}$ and NT flow regions, which can be defined as regions with and without vorticity, respectively (Westerweel et al. 2005).

$\dagger$ Email address for correspondence: carlos.silva@ist.utl.pt 
Whereas the vorticity and other quantities e.g. passive scalar concentration, display abrupt changes across the TNTI layer (Watanabe et al. 2014; Silva \& da Silva 2017), other quantities evolve smoothly across this layer e.g. the mean and fluctuating velocity fields vary slowly across the TNTI layer, as shown in Taveira \& da Silva (2013). In particular, while the cross Reynolds stresses, $\left\langle u_{i} u_{j}\right\rangle$, with $i \neq j$, are rigorously zero in the NT flow region near the TNTI layer, the normal Reynolds stresses, $\left\langle u_{i} u_{j}\right\rangle$ with $i=j$, are typically very high in this region. For example, Westerweel et al. (2009); Taveira \& da Silva (2013) have shown that the streamwise normal Reynolds stresses in the TNTI layer from a planar turbulent jet are already roughly one half their value inside the turbulent core region of the flow.

The fact that very important potential velocity fluctuations exist in the NT region close to the TNTI layer can be understood by the presence of both large and small scale vorticity structures in the $\mathrm{T}$ region bounding the TNTI layer. Indeed, it has been shown that these structures themselves define the geometry of the TNTI layer (da Silva et al. 2014) and the intense potential velocity fluctuations observed in the NT region can be explained by the motion induced by these structures (da Silva et al. 2011; Watanabe et al. 2017).

The investigation of the potential velocity fluctuations bounding a TNTI layer began with the pioneering work of Phillips (1955). Here we denote the fluctuating velocity vector field by $u_{i}(\vec{x}, t)$, with $i=1,2,3$, where $\vec{x}=x_{i}(i=1,2,3)$ and $t$ are the three spatial coordinates and the time, respectively, the index $i=2$ corresponds to the direction normal to the TNTI layer. Here use is made of the Reynolds decomposition i.e. the total velocity field is the sum of the mean and fluctuating velocity fields, respectively, $\left\langle U_{i}(\vec{x})\right\rangle+u_{i}(\vec{x}, t)$, and the brackets ' \langle\rangle ' represent an averaging operation to be described later. By using a kinetic energy spectrum with an infrared (low wave number) region of the form $E(k) \sim k^{4}$, which is implied by assuming that $E(k)$ is differentiable at the origin, Phillips (1955) was able to obtain asymptotic laws governing the profiles of all the second order moments of the fluctuating velocity $\left\langle u_{i} u_{j}\left(x_{2}\right)\right\rangle(i, j=1,2,3)$, as a function of the distance from the TNTI layer, $x_{2}$, provided this distance is much bigger than the integral scale of turbulence, $x_{2}>>L$ e.g. $\left\langle u_{1}^{2}\right\rangle \sim x_{2}^{-4}$. He was also able to demonstrate other interesting results e.g. $\left\langle u_{2}^{2}\left(x_{2}\right)\right\rangle=\left\langle u_{1}^{2}\left(x_{2}\right)\right\rangle+\left\langle u_{3}^{2}\left(x_{2}\right)\right\rangle$.

By using rapid distortion theory (RDT) Carruthers \& Hunt (1986) were able to extend the relation derived by Phillips (1955) for all the range of coordinates $x_{2}$, which allowed them to obtain the mean profiles of many important quantities in the NT region, such as the profile of the integral scale of turbulence. The analysis assumes that the NT region evolves in the absence of mean shear, and although the work focuses on the effects of stable stratification, the classical Phillips (1955) results are recovered when considering no stratification. More recently, Teixeira \& da Silva (2012) used RDT to extend the analysis of the potential velocity fluctuations by considering the effects of a thin viscous layer roughly coinciding with the TNTI layer. While extending the results obtained by Phillips (1955), both Carruthers \& Hunt (1986) and Teixeira \& da Silva (2012) recover the asymptotic results from Phillips (1955), such as the relation $\left\langle u_{2}^{2}\left(x_{2}\right)\right\rangle=$ $\left\langle u_{1}^{2}\left(x_{2}\right)\right\rangle+\left\langle u_{3}^{2}\left(x_{2}\right)\right\rangle$, which is independent of the shape of the kinetic energy spectrum assumed in the derivations.

The asymptotic laws governing the potential velocity fluctuations derived by Phillips (1955) have been also observed in a number of experimental works such as in turbulent jets (Bradbury 1965; Sunyach \& Mathieu 1969), turbulent wakes (Thomas 1973; Fabris 1979; Antonia et al. 1987), turbulent mixing layers (Wygnanski \& Fiedler 1970), and also in turbulent boundary layers (Bradshaw 1967; Kovasznay et al. 1970).

Numerical verification was also obtained in several works such as in turbulent planar 
jets (Taveira \& da Silva 2013). Surprisingly, all these works claim to have recovered the asymptotic relations $\left\langle u_{1}^{2}\right\rangle,\left\langle u_{2}^{2}\right\rangle,\left\langle u_{3}^{2}\right\rangle \sim x_{2}^{-4}$ however, it is important to stress that this law, like other similar laws derived by Phillips (1955), is based on the assumed form of the kinetic energy spectrum in the infrared region $\left(E(k) \sim k^{4}\right)$, also denoted as Batchelor spectrum (Davidson 2004), while, with the exception of Bradshaw (1967), no mention of the form of the spectra in the infrared region is made in the experiments.

It is noteworthy that Phillips (1955), Bradshaw (1967), Carruthers \& Hunt (1986) and Teixeira \& da Silva (2012) all mention the fact that their theoretical results for the irrotational velocity fluctuations depend on the form of the kinetic energy spectrum at low wave numbers, but they do not try to extend their results to different forms of the spectra. Nevertheless, it has been shown that spectra of the form $E(k) \sim k^{2}$ (Saffman 1967), also denoted as Saffman spectrum (Davidson 2004), do arise in several turbulent flows. Spectra with the general form $E(k) \sim k^{n}$, in the infrared region, where $n$ is a constant, have also been assumed to exist in several other turbulent flows (Birkhoff 1954; Eyink \& Thomson 2000; Vassilicos 2011; Oberlack \& Zieleniewicz 2013).

The present work uses rapid-distortion theory (RDT) to generalise the analytical results obtained by Phillips (1955), by considering turbulent flows where the kinetic energy spectrum in the infrared region follows the law $E(k) \sim k^{2}$ (Saffman turbulence). For this case, and for large distances from the TNTI $\left(x_{2}>>L\right)$, the velocity variance in the NT region evolves as $\left\langle u_{i}^{2}\right\rangle \sim x_{2}^{-3}(i=1,2,3)$, while the Taylor micro-scale $\lambda$, and viscous dissipation rate $\varepsilon$ evolve as $\langle\lambda\rangle \sim x_{2}$ and $\langle\varepsilon\rangle \sim x_{2}^{-5}$, respectively.

The new theoretical results are confirmed using several new direct numerical simulations (DNS) of shear-free turbulence (SFT), where a TNTI interface layer is generated in the absence of mean shear. Moreover, the effects the Reynolds number and peak wave number are assessed and it is shown that these results are robust and independent of these parameters. The new results are expected to be valid for other flow configurations e.g. in the presence of mean shear such as jets and wakes provided the kinetic energy spectra in the turbulence region exhibits a Batchelor or a Saffman spectrum, since, as described above, the results from Phillips (1955), which correspond to a Batchelor spectrum, have been observed in numerous flow configurations exhibiting mean shear.

This paper is organised as follows. The next section (Section 2) reviews the classical results obtained by Phillips (1955) and extends them using RDT by considering an infrared kinetic energy spectrum with a form $E(k) \sim k^{2}$. Section 3 describes the numerical methods, simulations and validation of the new simulations used in the present work. Section 4 assesses the new theoretical results obtained in this work. The paper ends with an overview of the main results of the paper (Section 5).

\section{Analytical descriptions of the irrotational flow region}

In this section the classical theoretical results derived by Phillips (1955) for the behaviour of the velocity fluctuations in the NT region near a TNTI layer are quickly reviewed and, by using rapid distortion theory (RDT) these results are extended for the case of a energy spectra obeying a power law of the form $E(k) \sim k^{2}$, in the infrared region.

\subsection{Phillips's asymptotic decay rates for the velocity fluctuations in the NT region}

In the seminal work by Phillips (1955) the problem of determining the velocity fluctuations in the irrotational flow region in the vicinity of a turbulent region was approached by considering potential inviscid flow. One of the boundaries of the quiescent fluid domain was set as an infinite perturbation plane, with a fluctuating velocity field 
defined as to satisfy a prescribed kinetic energy spectrum. Given the symmetry of the problem, tangential directions to the perturbation plane $\left(x_{1}\right.$ and $\left.x_{3}\right)$ are considered infinite. Phillips (1955) thus created a model for the TNTI where the effects of the perturbation plane in the irrotational flow domain are set by matching the normal velocity fluctuations at that plane. This model only allows for kinematic coupling between turbulent and non-turbulent regions i.e. it precludes the prescription of any type of dynamical interaction between the two flow regions.

Phillips (1955) then shows that as the distance from the TNTI $\left(x_{2}\right)$ increases, the importance of larger wave numbers is suppressed, so that the velocity fluctuations depend only on the shape of the infrared region of the spectrum at $x_{2}=0$. Specifically, the kinetic energy of the potential velocity fluctuations at a distance $x_{2}$ from the TNTI is (Phillips $1955)$

$$
\frac{1}{2}\left\langle\mathbf{u}^{2}\left(x_{2}\right)\right\rangle=\int \theta\left(k_{1}, k_{3}\right) e^{-2 k_{13} x_{2}} d k_{1} d k_{3}
$$

where $k_{13}=\sqrt{k_{1}^{2}+k_{3}^{2}}$ is the magnitude of wave number vector $\mathbf{k}_{\mathbf{1 3}}=\left(k_{1}, k_{3}\right)$ in planes $x_{2}=$ const., and $\theta\left(k_{1}, k_{3}\right)$ is a two-dimensional spectrum of the normal velocity component $u_{2}\left(x_{1}, 0, x_{3}\right)$ defined by,

$$
\theta\left(k_{1}, k_{3}\right)=\frac{1}{(2 \pi)^{2}} \int\left\langle u_{2}\left(x_{1}, 0, x_{3}\right) u_{2}\left(x_{1}^{\prime}, 0, x_{3}^{\prime}\right)\right\rangle e^{-i \mathbf{k}_{13} \cdot \mathbf{r}} d \mathbf{r}
$$

with, $\mathbf{r}=\left(r_{1}, r_{3}\right)=\left(x_{1}^{\prime}-x_{1}, x_{3}^{\prime}-x_{3}\right)$. The two-dimensional kinetic energy spectrum at a distance $x_{2}$ from the TNTI is given by,

$$
E\left(k_{13}, x_{2}\right)=\int \theta\left(k_{1}, k_{3}\right) e^{-2 k_{13} x_{2}} d S\left(k_{13}\right)=E_{0}\left(k_{13}\right) e^{-2 k_{13} x_{2}}
$$

where $d S\left(k_{13}\right)$ is the arc for the integration over circular shells with radius $k_{13}$, while $E_{0}\left(k_{13}\right)$ is the kinetic energy spectrum at the interface $\left(x_{2}=0\right)$. In order to compute the irrotational velocity fluctuations Phillips (1955) uses a spectrum $\theta\left(k_{1}, k_{3}\right)$ with the following form,

$$
\theta\left(\mathbf{k}_{\mathbf{1 3}}\right)=k_{i} k_{j} \theta^{i j}+O\left(k_{13}^{3}\right)
$$

where $\theta^{i j}$ is a constant, which is reminiscent of the usual form of the three-dimensional kinetic energy spectrum given by (Batchelor 1953),

$$
E(k)=C k^{4}+O\left(k^{6}\right)
$$

where $k=\left(k_{1}^{2}+k_{2}^{2}+k_{3}^{3}\right)^{1 / 2}$ is the magnitude of the three-dimensional wave number vector, $\mathbf{k}=\left(k_{1}, k_{2}, k_{3}\right)$, and $C$ is a constant. By replacing (2.4) into (2.1) Phillips (1955) arrives at,

$$
\frac{1}{2}\left\langle\mathbf{u}^{2}\right\rangle \sim x_{2}^{-4}
$$

which is then generalised into,

$$
\left\langle u_{i}^{2}\right\rangle \sim x_{2}^{-4}, \text { for } i=1,2 \text {, or } 3 .
$$

Phillips (1955) showed also that the potential velocity fluctuations obey the following relation,

$$
\left\langle u_{2}^{2}\left(x_{2}\right)\right\rangle=\left\langle u_{1}^{2}\left(x_{2}\right)\right\rangle+\left\langle u_{3}^{2}\left(x_{2}\right)\right\rangle
$$


and that all the cross stresses are zero e.g. $\left\langle u_{1} u_{2}\left(x_{2}\right)\right\rangle=0$. Additionally, using relations independent of the shape of the energy spectra (Phillips 1955; Teixeira \& da Silva 2012),

$$
\left\langle\left(\frac{\partial u_{i}}{\partial x_{1}}\right)^{2}\right\rangle=\left\langle\left(\frac{\partial u_{i}}{\partial x_{2}}\right)^{2}\right\rangle+\left\langle\left(\frac{\partial u_{i}}{\partial x_{3}}\right)^{2}\right\rangle,
$$

and,

$$
\left\langle\left(\frac{\partial u_{1}}{\partial x_{i}}\right)^{2}\right\rangle=\left\langle\left(\frac{\partial u_{2}}{\partial x_{i}}\right)^{2}\right\rangle+\left\langle\left(\frac{\partial u_{3}}{\partial x_{i}}\right)^{2}\right\rangle
$$

together with,

$$
\varepsilon=\nu\left[\left\langle\omega^{2}\right\rangle+2 \frac{\partial^{2}\left\langle u_{2}^{2}\right\rangle}{\partial x_{2}^{2}}\right]
$$

where $\nu$ is the kinematic viscosity and $\left\langle\omega^{2}\right\rangle$ is the mean enstrophy in the turbulence region, can be used to obtain the following scaling laws (inside the irrotational region), for the Taylor micro-scale,

$$
\lambda_{i} \sim x_{2},(i=1,2,3)
$$

defined as

$$
\lambda_{i}=\frac{\left\langle u_{i}^{2}\right\rangle}{\left\langle\left(\partial u_{i} / \partial x_{i}\right)^{2}\right\rangle}(\text { no summation })(i=1,2,3),
$$

and viscous dissipation,

$$
\varepsilon \sim x_{2}^{-6}
$$

This completes the scaling relations obtained by Phillips (1955) while assuming the form of the two-dimensional spectrum of the normal velocity component described in Eq. (2.4).

The dynamical interaction between the turbulent and non-turbulent flow regions, not accounted for in Phillips (1955), is included in Carruthers \& Hunt (1986), where pressure interactions are considered in a stably stratified non-turbulent region, and by Teixeira \& da Silva (2012), where viscous effects in the vicinity of the TNTI were included. These works resorted to rapid distortion theory (RDT), which is valid for time intervals much smaller than the characteristic time scale of non-linear interactions. The introduction of dynamical interactions between the turbulent and non-turbulent regions, by coupling the $\mathrm{T}$ and NT regions in a physically consistent way, preserves the asymptotic results at large distances from the interface, typically for distances from the TNTI larger than the integral scale of turbulence $x_{2}>>L$ (Carruthers \& Hunt 1986; Teixeira \& da Silva 2012). Similarly, Teixeira \& da Silva (2012) show that the effects of viscosity, which are restricted in that case to a very thin layer within the TNTI, quickly fade away from this interface, in agreement with Eq. (2.1) from Phillips (1955), which implies that the velocity fluctuations far away from the TNTI depend only on the largest scales of the flow within the turbulent region.

\subsection{Extending the classical results for an infrared energy spectrum with $E(k) \sim k^{2}$}

As mentioned in the introduction, while Phillips (1955); Bradshaw (1967); Carruthers \& Hunt (1986); Teixeira \& da Silva (2012) mention the dependency of the asymptotic behaviour laws for the irrotational velocity fluctuations on the shape of the kinetic energy spectrum at lower wave numbers, they did not extend their results for a general kinetic energy spectrum of the form $E(k) \sim k^{n}$, even though turbulent flows with $n=4$ (Batchelor turbulence) and $n=2$ (Saffman turbulence), as well as other cases, have 
been reported (Davidson 2004). This generalisation for the case $n=2$ is done in this section.

The expression for the variance of the tangential velocity fluctuations in the irrotational part of the flow was given by Teixeira \& da Silva (2012) (their equation (3.18)) as:

$$
\left\langle u_{1}^{2}\left(x_{2}\right)\right\rangle=\frac{1}{16 \pi} \int_{0}^{2 \pi} \int_{0}^{+\infty} \int_{-\infty}^{+\infty} \frac{E(k)}{k^{4}} k_{13}^{3} \cos ^{2} \alpha e^{-2 k_{13} x_{2}} d k_{2} d k_{13} d \alpha,
$$

where $k_{13}=\left(k_{1}^{2}+k_{3}^{2}\right)^{1 / 2}, k_{1}$ and $k_{3}$ are the components of the wavenumber in the directions tangential to the TNTI, and $k_{2}$ is the component of the wavenumber in the normal direction. $\alpha$ is the angle made by $\left(k_{1}, k_{3}\right)$. Note that in $(2.15)$ cylindrical coordinates were adopted for the integrals. This expression is valid for any energy spectrum $E(k)$, with $k=\left(k_{1}^{2}+k_{2}^{2}+k_{3}^{2}\right)^{1 / 2}=\left(k_{13}^{2}+k_{2}^{2}\right)^{1 / 2}$.

In Teixeira \& da Silva (2012), an exponential energy spectrum with a power law of $k^{4}$ in the infrared region was considered, namely:

$$
E(k)=\frac{1}{(2 \pi)^{1 / 2}} q^{2} \lambda_{\infty}\left(k \lambda_{\infty}\right)^{4} e^{-\frac{1}{2}\left(k \lambda_{\infty}\right)^{2}}
$$

(their equation (2.26)), where $q$ is the RMS velocity in the homogeneous turbulence to the other side of the T/NT interface, and $\lambda_{\infty}$ is the corresponding Taylor microscale. An energy spectrum of the same type, but where the power law in the infrared region is $k^{2}$, takes the form:

$$
E(k)=\frac{9}{5}\left(\frac{3}{10 \pi}\right)^{1 / 2} q^{2} \lambda_{\infty}\left(k \lambda_{\infty}\right)^{2} e^{-\frac{3}{10}\left(k \lambda_{\infty}\right)^{2}} .
$$

Note that both the coefficient that defines the amplitude of the spectrum and that in the exponential have different values from those in (2.16), because the spectrum must be consistent with the definitions of RMS velocity and Taylor microscale. Another property of the exponential spectra of the form (2.17) is that their integral length scale $L_{\infty}$ and Taylor microscale $\lambda_{\infty}$ are related by a fixed factor. For Eq. (2.16), the relation is $L_{\infty}=(\pi / 2)^{1 / 2} \lambda_{\infty}$ (as noted by Teixeira \& da Silva 2012), and for Eq. (2.17) $L_{\infty}=(27 \pi / 40)^{1 / 2} \lambda_{\infty}$.

If $(2.17)$ is used in (2.15), the integration over the angle $\alpha$ may be performed analytically, because $E(k)$ does not depend on $\alpha$, yielding multiplication by a factor of $\pi$. The integration over the wavenumber $k_{2}$ is less immediate, yielding:

$$
\left\langle u_{1}^{2}\left(x_{2}\right)\right\rangle=\frac{9}{80}\left(\frac{3 \pi}{10}\right)^{1 / 2} q^{2} \int_{0}^{+\infty}\left[1-\Phi\left(\sqrt{\frac{3}{10}} k_{13}^{\prime}\right)\right] k_{13}^{\prime 2} e^{-2 k_{13}^{\prime}\left(x_{2} / \lambda_{\infty}\right)} d k_{13}^{\prime},
$$

where $k_{13}^{\prime}=k_{13} \lambda_{\infty}$ and $\Phi$ is the error function. This expression is considerably more complicated than that obtained by Teixeira \& da Silva (2012) for (2.16), but its asymptotic behaviour as $x_{2} \rightarrow \infty$ can be obtained in a similar way, affording some simplifications. Namely, the contributions to the integral over $k_{13}$ when $x_{2} \gg 1$ come mainly from low values of $k_{13}$. When this approximation is made, the error function reduces approximately to zero, and (2.18) simplifies to:

$$
\left\langle u_{1}^{2}\left(x_{2}\right)\right\rangle \sim \frac{9}{320}\left(\frac{3 \pi}{10}\right)^{1 / 2} \frac{q^{2}}{\left(x_{2} / \lambda_{\infty}\right)^{3}} .
$$

Note that the proportionality coefficients present in this expression depend on the shape of the spectrum (2.17), more specifically on the constants included in its definition, but the type of asymptotic variation with $x_{2}$ only relies on the type of dependence of the 
spectrum at low wavenumbers. That was also the case with the asymptotic expression obtained by Teixeira \& da Silva (2012) for a spectrum proportional to $k^{4}$ in the infrared region, which yielded instead:

$$
\left\langle u_{1}^{2}\left(x_{2}\right)\right\rangle \sim \frac{3}{128} \frac{q^{2}}{\left(x_{2} / \lambda_{\infty}\right)^{4}}
$$

(their equation (3.20)). Since the relation stating that $\left\langle u_{2}^{2}\left(x_{2}\right)\right\rangle=2\left\langle u_{1}^{2}\left(x_{2}\right)\right\rangle$ remains valid for any energy spectrum, it is straighforward to obtain $\left\langle u_{2}^{2}\left(x_{2}\right)\right\rangle$ from $(2.19)$ (or $(2.20)$ ).

By using the relations presented in section 2.1, the asymptotic power law governing the viscous dissipation rate can be easily obtained:

$$
\varepsilon=2 \nu \frac{\partial^{2}\left\langle u_{2}^{2}\right\rangle}{\partial x_{2}^{2}} \sim \frac{27}{20}\left(\frac{3 \pi}{10}\right)^{1 / 2} \nu \frac{q^{2}}{\lambda_{\infty}^{2}} \frac{1}{\left(x_{2} / \lambda_{\infty}\right)^{5}}=\frac{9}{100}\left(\frac{3 \pi}{10}\right)^{1 / 2} \frac{\varepsilon_{\infty}}{\left(x_{2} / \lambda_{\infty}\right)^{5}},
$$

where, in the last equality, the fact that the dissipation rate in the isotropic turbulence is defined as $\varepsilon_{\infty}=15 \nu q^{2} / \lambda_{\infty}^{2}$ has been used. This should be compared with the corresponding relation obtained by Teixeira \& da Silva (2012), which can be written:

$$
\varepsilon=\frac{1}{8} \frac{\varepsilon_{\infty}}{\left(x_{2} / \lambda_{\infty}\right)^{6}}
$$

(their Eq. (3.22)).

The Taylor microscale is defined as (see Eq. (3.27) of Teixeira \& da Silva (2012)):

$$
\lambda_{1}=\left[\frac{\left\langle u_{1}^{2}\right\rangle}{\left\langle\left(\frac{\partial u_{1}}{\partial x_{1}}\right)^{2}\right\rangle}\right]^{1 / 2} .
$$

Its behaviour is controlled by the behaviour of $\left\langle\left(\partial u_{1} / \partial x_{1}\right)^{2}\right\rangle$ (in addition to that of $\left\langle u_{1}^{2}\right\rangle$ ). Since differentiation with respect to $x_{1}$ corresponds in Fourier space to multiplication by $k_{1}$, by analogy with $(2.15),\left\langle\left(\partial u_{1} / \partial x_{1}\right)^{2}\right\rangle$ can be expressed as

$$
\left\langle\left(\frac{\partial u_{1}}{\partial x_{1}}\right)^{2}\right\rangle=\frac{1}{16 \pi} \int_{0}^{2 \pi} \int_{0}^{+\infty} \int_{-\infty}^{+\infty} \frac{E(k)}{k^{4}} k_{13}^{5} \cos ^{4} \alpha e^{-2 k_{13} x_{2}} d k_{2} d k_{13} d \alpha .
$$

Following the same procedure as before, the integration over $\alpha$ is straightforward, corresponding to multiplication by $3 \pi / 4$, and the integration over $k_{2}$ proceeds identically as in (2.15), using also (2.17). Taking again the limit $x_{2} \rightarrow \infty$, for which contributions to the integral come mostly from small $k_{13}$, yields:

$$
\left\langle\left(\frac{\partial u_{1}}{\partial x_{1}}\right)^{2}\right\rangle \sim \frac{81}{1280}\left(\frac{3 \pi}{10}\right)^{1 / 2} \frac{q^{2}}{\lambda_{\infty}^{2}} \frac{1}{\left(x_{2} / \lambda_{\infty}\right)^{5}} .
$$

Then, using the definition (2.23), and also (2.25) and (2.19), the Taylor microscale can be shown to take the asymptotic form for $x_{2} \rightarrow \infty$ :

$$
\lambda_{1} \sim \frac{2}{3} x_{2}
$$

This result shows that the Taylor microscale increases linearly from the TNTI interface into the irrotational flow region, having the same qualitative asymptotic behaviour for the energy spectrum (2.17) as the expression obtained by Teixeira \& da Silva (2012) for the energy spectrum (2.16), namely:

$$
\lambda_{1} \sim\left(\frac{4}{15}\right)^{1 / 2} x_{2}
$$




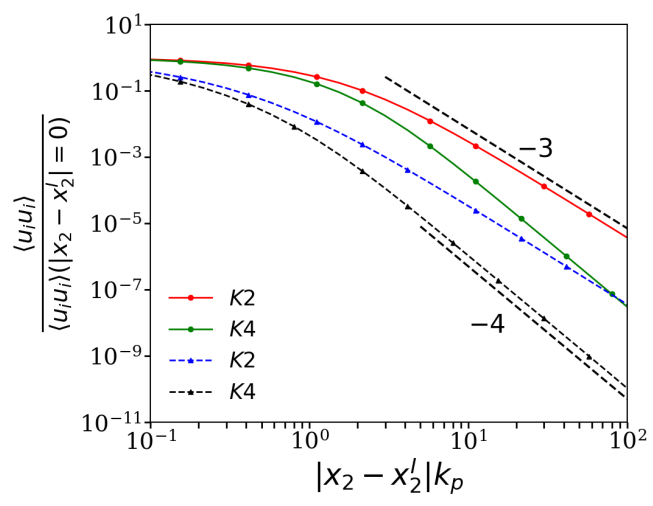

Figure 1: Profiles of $\left\langle u_{i} u_{i}\right\rangle$ as function of the distance from the TNTI layer $\left(x_{2}\right)$ obtained though numerical integration of Eq. (2.31) by using the initial spectrum used in the DNS (Eq. 3.1) - solid lines - and the one defined in Eq. (2.28) - dashed lines, for a Batchelor spectrum $(n=4)$ and a Saffman spectrum $(n=2)$. In all cases $k_{p}=80$, $k_{\eta}=8 / 3 \times 10^{5}$. The mean profiles of kinetic energy $\left\langle u_{i} u_{i}\right\rangle$ are normalised by their value at the irrotational boundary (IB) which is the surface separating the turbulent and non-turbulent flow regions, and is located at $x_{2}= \pm x_{2}^{I}$, while the distance from the IB $\left(x_{2}-x_{2}^{I}\right)$, is normalised by the integral scale of turbulence assumed to be $L=k_{p}^{-1}$.

(their equation (3.29))

\subsection{Effect of the form of the kinetic energy spectrum in the new theoretical results}

It is well known that the shape of the initial kinetic energy spectrum largely determines the temporal evolution of decaying isotropic turbulence (Davidson 2004). Moreover, as described above, the spectrum also has a crucial effect on the statistics of the potential velocity fluctuations in the irrotational region near a TNTI, as shown in Eq. (2.15). However, it is often argued that the asymptotic behaviour of the velocity fluctuations in the NT region depend only on the infrared region of the kinetic energy spectrum, which is assumed to follow a power law $E(k) \sim k^{n}$ for $k<k_{p}$, because the higher wave numbers $k>k_{p}$ of the energy spectra are modulated by the increase in the distance to the TNTI as shown in Eq. (2.3). Therefore, it is important to insure that a particular shape of the kinetic energy spectra at high wave numbers $\left(k>k_{p}\right)$ does not affect the present theoretical results.

In order to assess this we employ a simple model spectrum defined by Pope (2000),

$$
E(k)=C_{k} \varepsilon^{2 / 3} f_{k_{p}} f_{k_{\eta}} k^{-\frac{5}{3}},
$$

where $C_{k}=1.5$ is the Kolmogorov constant, with,

$$
f_{k_{p}}=\left\{\frac{k / k_{p}}{\left[\left(k / k_{p}\right)^{2}+c_{k_{p}}\right]^{1 / 2}}\right\}^{\frac{5}{3}+n},
$$

and,

$$
f_{k_{\eta}}=\exp \left\{-\beta\left[\left[\left(k / k_{\eta}\right)^{4}+c_{k_{\eta}}^{4}\right]^{1 / 4}-c_{k_{\eta}}\right]\right\}
$$


where, $k_{p}$ is again the peak wave number, $k_{\eta}$ is a dissipation scale wave number, and the parameters $\beta, c_{k_{p}}$ and $c_{k_{\eta}}$ are chosen for the spectrum to represent fully developed isotropic turbulence at high turbulent Reynolds number $R e_{\lambda}$. Specifically, these parameters were set to $\beta=5.2, c_{k_{p}}=6.78$ and $c_{k_{\eta}}=0.40$ (Pope 2000).

The model spectrum defined by Eq. (2.28) allows assessing the dependence of the asymptotic mean profiles of kinetic energy on the integral length scale of turbulence, which is assumed to be $L=k_{p}^{-1}$, and also on the power law of the infrared kinetic energy spectrum $n$. Moreover, by using distinct values of the ratio $k_{\eta} / k_{p}$ the dependence of the results on $R e_{\lambda}$ can be explored.

We start this analysis by comparing the results obtained from integrating the following equation,

$$
\left\langle u_{1}^{2}\left(x_{2}\right)\right\rangle=\frac{1}{16} \int_{0}^{\pi} \int_{0}^{+\infty} E(k) \sin ^{3} \theta e^{-2 k \sin \theta x_{2}} d k d \theta,
$$

which is the same as Eq. (2.15) but with the integral expressed in spherical polar coordinates, instead of cylindrical coordinates (Teixeira \& da Silva 2012), using i) the form of the initial energy spectrum employed in the DNS of DHIT - Eq. (3.1) shown in the next section, and $i$ ) the spectrum given by Eq. (2.28) reproducing a Batchelor $(n=4)$ or a Saffman $(n=2)$ spectrum.

Figure 1 shows the resulting profiles of $\left\langle u_{i} u_{i}\right\rangle$ as function of the distance $\left(x_{2}-x_{2}^{I}\right)$ from a particular surface within the TNTI layer - the so-called irrotational boundary (IB) which is the surface separating the turbulent and non-turbulent flow regions, which is located at $x_{2}= \pm x_{2}^{I}$. The profiles of $\left\langle u_{i} u_{i}\right\rangle$ have been obtained by integrating Eq. (2.31) using the two expressions for $E(k)$ i.e. using Eq. (2.28) and Eq. (3.1). For the integration of Eq.(2.28) we prescribed $k_{p}=80$ and $k_{\eta}=8 / 3 \times 10^{5}$. It is clear that no difference is observed in the resulting power laws for the kinetic energy decay rate in both cases, since we recover $\left\langle u_{i} u_{i}\right\rangle \sim x_{2}^{-3}$ and $\left\langle u_{i} u_{i}\right\rangle \sim x_{2}^{-4}$ for $n=2$ and $n=4$, respectively, which is equal to the asymptotic power laws obtained when $E(k) \sim k^{n}$ is used to analytically integrate Eq. (2.31).

Figure 2 shows the effects of the turbulent Reynolds number on the kinetic energy decay rates in the non-turbulent flow region. Notice that this is equivalent to assessing the effects of the size of inertial range region of the kinetic energy spectrum $k_{p}<k<k_{\eta}$. Figure 2a shows the spectra given by Eq. (2.28) while figure $2 \mathrm{~b}$ shows the profiles of $\left\langle u_{i} u_{i}\right\rangle$ in the non-turbulent region resulting from numerical integration of these spectra by solving Eq. (2.31). Different input parameters are used for the spectra. We fix $k_{p}=80$ and vary the dissipation wave number by using $k_{\eta}=1 / 3 \times 10^{4}, 8 / 3 \times 10^{5}$ and $8 / 3 \times 10^{6}$, which correspond to $R e_{\lambda} \sim 31,576$ and 2674 respectively. The Reynolds number $R e_{\lambda}$ was estimated by taking $k_{p} \approx L^{-1}, k_{\eta} \approx \eta^{-1}$ and by using the following relations for isotropic turbulence (Pope 2000),

$$
\frac{k_{p}}{k_{\eta}} \sim \frac{\eta}{L} \approx R e_{L}^{-\frac{3}{4}}
$$

and,

$$
R e_{\lambda}=\left(\frac{20}{3} R e_{L}\right)^{\frac{1}{2}} .
$$

For low Reynolds numbers $\left(R e_{\lambda} \sim 31\right)$, which are similar to the corresponding DNS used in the present work, the form of the spectrum resembles the one used to start the present DNS of decaying homogeneous isotropic turbulence (DHIT), while the other cases clearly resemble spectra from high Reynolds number turbulence, where the values 


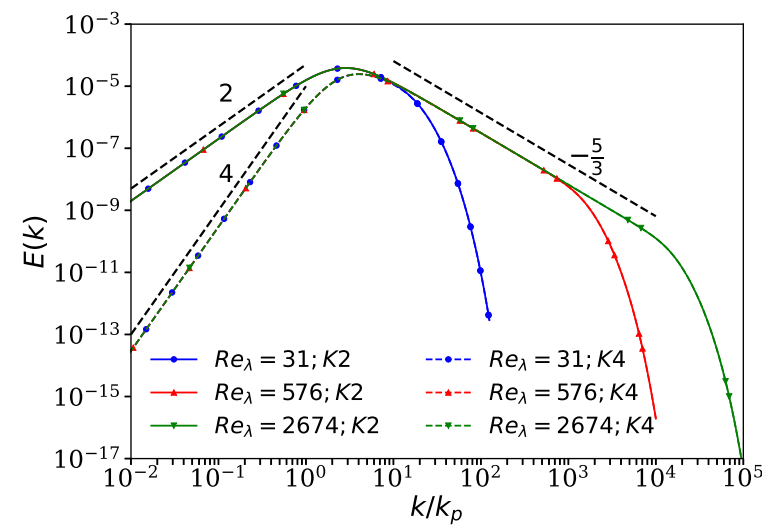

(a) $E(k)$ defined by Eq. (2.28).

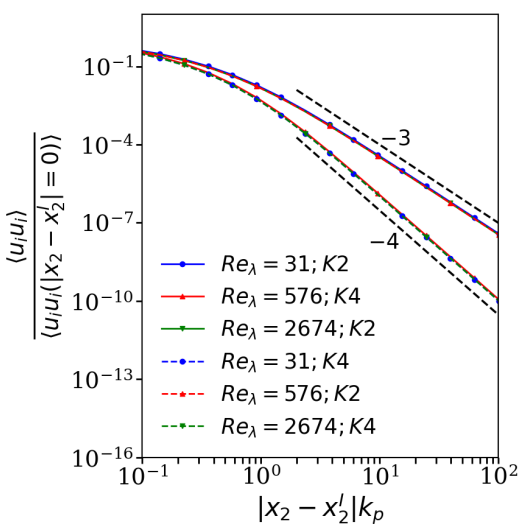

(b) $\left\langle u_{i} u_{i}\left(x_{2}\right)\right\rangle$ obtained by integrating Eq. (2.31).

Figure 2: Effect of the Reynolds number, represented by the size of the wave number range between $k_{p}<k<k_{\eta}$, and the profiles of $\left\langle u_{i} u_{i}\right\rangle$ in the non-turbulent region obtained by integrating Eq. (2.31) with the spectra given by Eq. (2.28) with varying input parameters, for both $n=2$ and $n=4$. All spectra have $k_{p}=80$, where solid and dashed lines correspond to $n=2$ and $n=4$, respectively. The values of $k_{\eta}$ used are $1 / 3 \times 10^{4}, 8 / 3 \times 10^{5}$ and $8 / 3 \times 10^{6}$, which correspond to $\operatorname{Re}_{\lambda}$ of 31,576 and 2674 respectively.

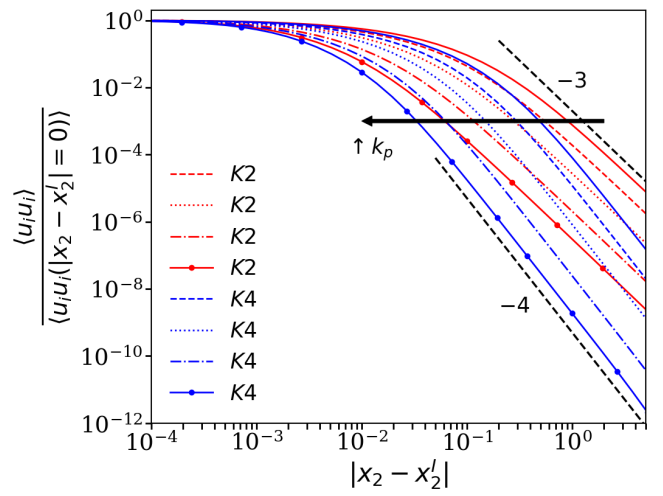

(a) Profiles of $\left\langle u_{i} u_{i}\right\rangle$ for several values of $k_{p}$.

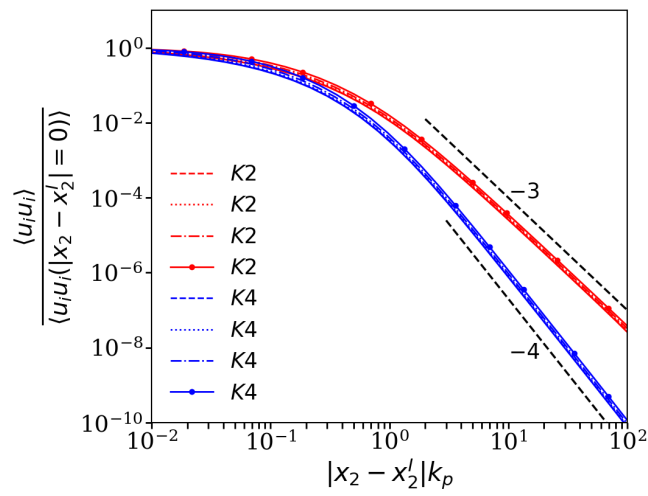

(b) Profiles of $\left\langle u_{i} u_{i}\right\rangle$ for several values of $k_{p}$, where $x_{2}$ is normalised by $k_{p}$.

Figure 3: Effect of the peak wave number $k_{p}$ on the profiles of $\left\langle u_{i} u_{i}\right\rangle$ in the non-turbulent region of the SFT simulations, obtained by integrating Eq. (2.31) with spectra given by Eq. (2.28), for both $n=2$ and $n=4$, where $k_{p}$ is varied between $k_{p}=3,5,10$, and 20: (a) using the normal distance $x_{2}$ without any normalisation (the arrow indicates increasing values of $k_{p}$ ); (b) normalising $x_{2}$ with the peak wave number $k_{p}$.

of $k_{\eta}$ were chosen to obtain $R e_{\lambda}$ differing by one order of magnitude (Pope 2000). It is clear that the magnitude of the Reynolds number, or equivalently the extent of the wave number region between $k_{p}<k<k_{\eta}$, does not alter the scaling laws for the non-turbulent profiles of $\left\langle u_{i} u_{i}\right\rangle$ obtained from Eq. (2.31). This result justifies the use DNS of DHIT 
with relatively low $R e_{\lambda}$ as a valid method to explore the asymptotic behaviour of the velocity fluctuations in the irrotational region.

The effect of the spectrum peak $k_{p}$ on the asymptotic decay laws of the kinetic energy in the non-turbulent region is investigated in figure 3 , showing the mean profiles of $\left\langle u_{i} u_{i}\right\rangle$ in the non-turbulent region resulting from the numerical integration of Eq. (2.31) using Eq. (2.28) as input, for several values of $k_{p}$, specifically, we use $k_{p}=3,5,10$, and 20 . As in the previous figure, both the cases $n=2$ and $n=4$ are considered. As the value of $k_{p}$ increases, and therefore the integral scale $L$ decreases, the power law behaviour in the profiles velocity variance $\left\langle u_{i} u_{i}\right\rangle$ begins closer to the interface $\left(x_{2}=0\right)$ (Fig. 3a). Interestingly, when the normal distance $x_{2}$ is normalised by $k_{p}$ all the mean profiles of $\left\langle u_{i}^{2}\right\rangle$ collapse (Fig. 3b). Since the integral length scale of turbulence is assumed to be $L \sim k_{p}^{-1}$ this shows that the power law appears whenever $x_{2}>L$, as expected, however, in no way does the prescribed value of $k_{p}$ affect the decay rate of the power laws $\left\langle u_{i} u_{i}\right\rangle \sim x_{2}^{-4}$ and $\left\langle u_{i} u_{i}\right\rangle \sim x_{2}^{-3}$ obtained for $n=4$ and $n=2$, respectively.

\section{Direct numerical simulations}

This section describes the numerical methods employed to carry out all the simulations analysed in the present work. The work relies on direct numerical simulations (DNS) of two flow configurations: i) decaying homogeneous isotropic turbulence (DHIT) and, ii) shear-free turbulence (SFT) where a turbulent front evolves into a quiescent (nonturbulent) flow region in the absence of mean shear.

\subsection{Numerical methods}

All the simulations employ the same code, which is an in-house Navier-Stokes solver that uses the classical pseudo-spectral methods (collocation method) for spatial discretization (Canuto et al. 1987), and a three-step 3rd-order explicit Runge-Kutta scheme for the temporal advancement (Williamson 1980). The simulation domain is a triple periodic cube with sides equal to $2 \pi$ and the simulations are fully de-aliased using the $2 / 3$ rule. The computational grid is uniform and isotropic $\left(\Delta x_{1}=\Delta x_{2}=\Delta x_{3}\right)$ with $N_{1} \times N_{2} \times N_{3}$ collocation points, where $N_{1}=N_{2}=N_{3}$. More details on this code can be found in Silva et al. (2018) and references therein.

\subsection{Direct numerical simulations of DHIT and SFT}

In the present work we have carried out two DNS of decaying homogeneous isotropic turbulence (DHIT). Both simulations use a total of $N_{1} \times N_{2} \times N_{3}=1024 \times 1024 \times 1024$ collocation points along the $x_{1}, x_{2}$, and $x_{3}$ directions, respectively, and the kinematic viscosity is equal to $\nu=2.67 \times 10^{-4}$.

The two simulations basically differ in the details of the initial velocity field which is obtained from a random number generator, that creates a divergent free velocity field with initial variance equal to $\left\langle u_{1}^{2}\right\rangle=\left\langle u_{2}^{2}\right\rangle=\left\langle u_{3}^{2}\right\rangle=1$, and with prescribed kinetic energy spectrum with the following form,

$$
E(k) \sim k^{n} e^{-2\left(\frac{k}{k_{p}}\right)^{2}},
$$

where $k_{p}$ defines the peak wave number while $n$ controls the slope in the low wave number (infrared) region (Ishida et al. 2006). We designate by $K 4$ and $K 2$ the two DHIT simulations, where the parameter $n$ was set to $n=4$ (Batchelor turbulence Batchelor (1953)) and $n=2$ (Saffman turbulence - Saffman (1967)), respectively, while the peak wave number is located at $k_{p}=30$, for both simulations. Figure 4 shows the 


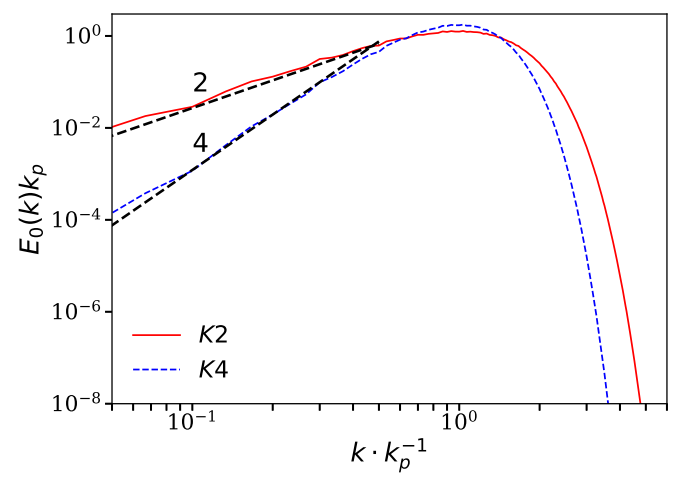

Figure 4: Initial kinetic energy spectrum for the two DNS of DHIT used in the present work. $K 2$ and $K 4$ correspond to the cases where the parameter $n$ in Eq. (3.1) was set to 2 and 4 , respectively.

initial kinetic energy spectrum for the two simulations, with the infrared slopes of $n=2$ and $n=4$, for $K 2$ and $K 4$, respectively.

As in Ishida et al. (2006), we define a Reynolds number based on the integral scale of turbulence which is assumed to be equal to the peak wave number $L=k_{p}^{-1}$. This Reynolds number is defined as $R e_{k_{p}}=\left\langle u^{2}\right\rangle^{1 / 2} /\left(k_{p} \nu\right)$, and similarly in the following discussion the time is normalised by the initial integral length scale turnover time $T_{k_{p}}=$ $1 /\left(\left\langle u^{2}\right\rangle^{1 / 2} k_{p}\right)$ i.e. $\tau=t / T_{k p}$. The initial Reynolds number for both simulations is equal to $R e_{k_{p}}=125$, as in the DHIT simulations of Ishida et al. (2006).

As shown in Ishida et al. (2006), in order to preserve the slope of the kinetic energy spectrum at low wave numbers during the kinetic energy decay phase, the initial spectrum peak must be $k_{p}>20$. Ishida et al. (2006) also show that in order to attain the correct (theoretical) kinetic energy decay rates in DNS of DHIT, the Reynolds number must be at least $R e_{k_{p}}>100$. These constraints are both fulfilled with the values of $R e_{k_{p}}=125$ and $k_{p}=30$ used in the present simulations. Moreover, the ratio of the maximum effective wave number $k_{\max }$, and the peak wave number is equal to $k_{\max } / k_{p}=12$. This choice allows maximizing the Reynolds number $R e_{k_{p}}$, while keeping the appropriate resolution. Starting with the initial conditions described above, each DHIT simulation follows the typical evolution described in many previous works (e.g. Ishida et al. (2006)). Initially enstrophy increases with time as the kinetic energy spectrum develops, increasing the range of active wave numbers, until it attains a maximum $\left(\tau_{\omega}\right.$ - enstrophy peak), which is then followed by a slow decay. As will be shown below for both simulations, the rates of kinetic energy decay $\left\langle u^{2}\right\rangle \sim \tau^{-m}$ recover the expected theoretical values, with $m=10 / 7$ and $m=6 / 5$ for the $K 4$ and $K 2$ simulations respectively, after the enstrophy peak is attained.

From these two DHIT simulations, we generate DNS of shear-free turbulence (SFT) where a turbulent front, separating turbulent and non-turbulent flow regions, evolves in the absence of mean shear. The procedure to obtain the SFT simulations has been described in several papers Perot \& Moin (1995); Cimarelli et al. (2015); Silva et al. (2018) and is here only briefly outlined.

Each SFT simulation starts from the velocity field obtained at a given time $\tau=\tau_{S F T}$, from one of the DHIT simulations, where $\tau_{S F T}$ is always taken at a time after the enstrophy peak, $\tau_{S F T}>\tau_{\omega}$. At the selected time instant of the desired DHIT simulation, 
the velocity in the central domain region $-H / 2 \leqslant x_{2} \leqslant H / 2$, is preserved, while the velocity in the remaining domain is set to zero, where $H$ is a parameter defining the width of the initial turbulent region. This is done through the convolution of the velocity field with a hyperbolic tangent profile (Cimarelli et al. 2015; Silva et al. 2018), which creates an initial SFT flow where turbulent $\left(H / 2 \leqslant x_{2} \leqslant H / 2\right)$ and non-turbulent $\left(x_{2}<\right.$ $-H / 2 \wedge x_{2}>H / 2$ ) flow regions are separated by a turbulent/non-turbulent interface. Each SFT then evolves for a few time iterations, whereby the initial isotropic turbulence flow region spreads into the irrotational (quiescent) region, in the absence of mean shear. Therefore, two distinct (upper and lower) TNTI layers develop within the computational domain, as the turbulence slowly decays within the core of the turbulent region.

Several widths of the initial turbulence region $H$ were tested, to determine the influence of the hyperbolic tangent profile convolution on the shape of the resulting kinetic energy spectra. It was found that the effects of the hyperbolic tangent convolution were minimised with $H / 2 \pi \approx 1 / 5$. It is important to maximize both the size of the initial turbulent region $H$, so that the large scales of motion remain unaffected, and the size of the non-turbulent region $(2 \pi-2 H)$ to insure that it is not affected by the (periodic) boundary conditions. Several numerical experiments then led to the optimal ratio $H / 2 \pi \approx 1 / 5$ used here. In this process we also confirmed the need to use a size of the turbulent region with at least $H \gtrsim 4 L$ as described in Cimarelli et al. (2015) (we are using $H>>4 L$ ). Moreover, the adaptation of the irrotational flow region was found to require only 4 time steps, which correspond to $1.84 \tau_{\eta}$ and $1.56 \tau_{\eta}$ for the simulations $K 2$ and $K 4$, respectively, where $\tau_{\eta}=(\nu / \varepsilon)^{1 / 2}$ is the Kolmogorov time, and $\varepsilon$ is the viscous dissipation rate.

The identification of the TNTI layer was done using the volume method described in Silva et al. (2018) and references therein, which characterises the irrotational boundary (IB), i.e. the outermost layer of the TNTI, by assessing the variation of the vorticity magnitude with the volume of the turbulent flow region. This allows the determination of a vorticity magnitude threshold $\omega_{t h}$ that identifies the IB.

Figure 5 shows the contours of enstrophy for the $K 4$ simulation at several instants corresponding to the SFT phase of the simulation. The initial size of the turbulent region contains many integral scales with $H \approx 38 L$ in both cases, and it is clear that the TNTI remains quite far from the boundaries of the computational domain. For the instants considered, few strong excursions of turbulent flow into the irrotational flow region can be observed, and the large scale geometry of the TNTI remains relatively flat. This allows one to use 'classical' statistics i.e. averaging any flow quantity at a given $x_{2}$ coordinate by using the two homogeneous flow directions $\left(x_{1}, x_{3}\right)$, instead of using the conditional statistics featured in many recent works dealing with the TNTI. This choice is also supported by many well-known classical results which have reported that the asymptotic results from Phillips (1955) can be easily observed using similar 'classic' averaging procedures e.g. (Bradshaw 1967).

Figure 6 shows a zoom of the TNTI for both simulations at approximately the same instant $(\tau \approx 30)$. The TNTI is sharp for both simulations, as observed in numerous other studies dealing with this interface, and it is clear that the turbulent region $H$ contains a large number of large scale structures.

\subsection{Assessment of the DHIT and SFT simulations}

Figure 7 shows the temporal evolution of the kinetic energy, $\frac{1}{2}\left\langle\mathbf{u}^{2}\right\rangle$, and enstrophy $\left\langle\omega^{2}\right\rangle=\left\langle\omega_{i} \omega_{i}\right\rangle$, for two DNS of DHIT. Initially, for times $\tau \lesssim 1$, the enstrophy and therefore also the kinetic energy dissipation rate $\varepsilon=\nu\left\langle\omega_{i} \omega_{i}\right\rangle$ (where $\omega_{i}=\varepsilon_{i j k} \partial u_{j} / \partial x_{k}$ is the vorticity vector) are negligible. However, for $\tau \gtrsim 1$ the enstrophy (and dissipation) 


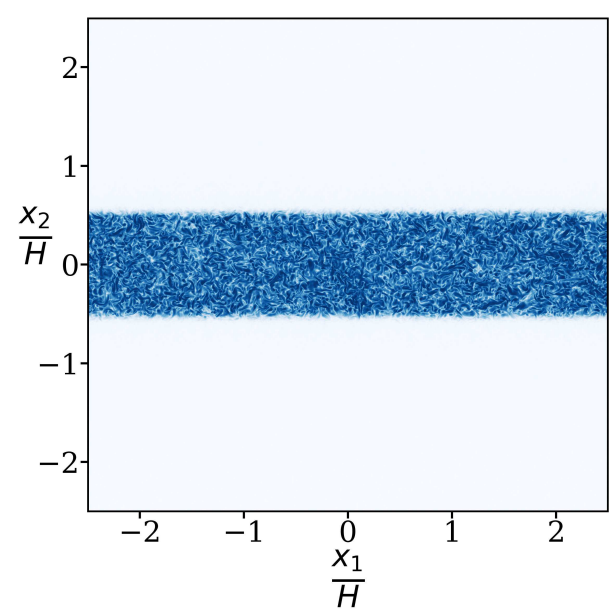

(a) $\tau=24.69$

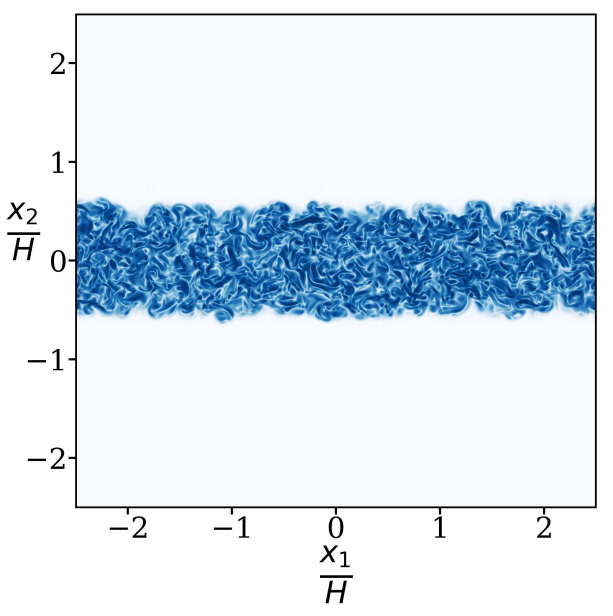

(c) $\tau=61.01$

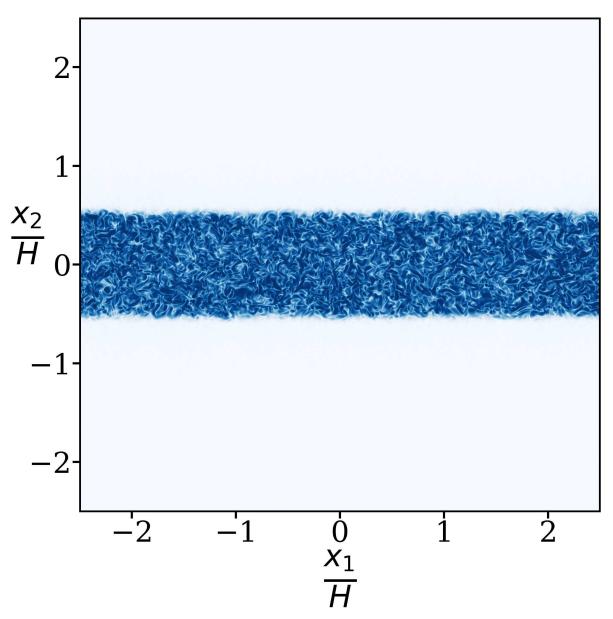

(b) $\tau=31.43$

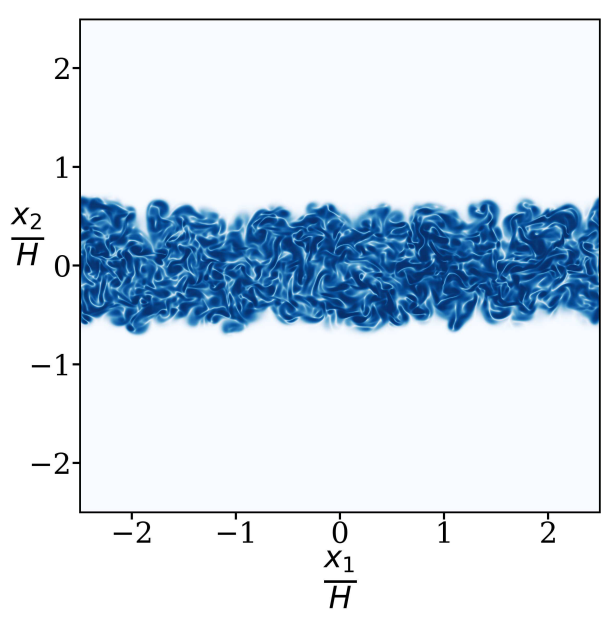

(d) $\tau=112.05$

Figure 5: Enstrophy contours in the $\left(x_{1}, x_{2}\right)$ plane for the $K 4$ simulation for several instants corresponding to the SFT phase of the simulation.

increase reaching a maximum at $\tau=\tau_{\omega}$ (enstrophy peak), which is followed by a decay in enstrophy (and dissipation). The kinetic energy decays from the start of the simulation, initially, very slowly $(\tau \lesssim 1)$, and attains its maximum decay rate roughly when the viscous dissipation is at its maximum $\left(\tau=\tau_{\omega} \approx 5\right)$, which is followed by a smaller decay rate $(\tau \gtrsim 10)$, where the classical theoretical laws for the decay of isotropic turbulence are recovered after $\tau \sim 70$.

The theoretical decay laws for the kinetic energy decay in isotropic turbulence for Batchelor turbulence (Batchelor 1953) and Saffman turbulence (Saffman 1967), corresponding to the simulations $K 4$ and $K 2$, respectively, can be attested in figure 8 . The figure shows the temporal evolution for the exponent $m$ for simulations $K 4$ and $K 2$, defining the rate of kinetic energy decay i.e. 


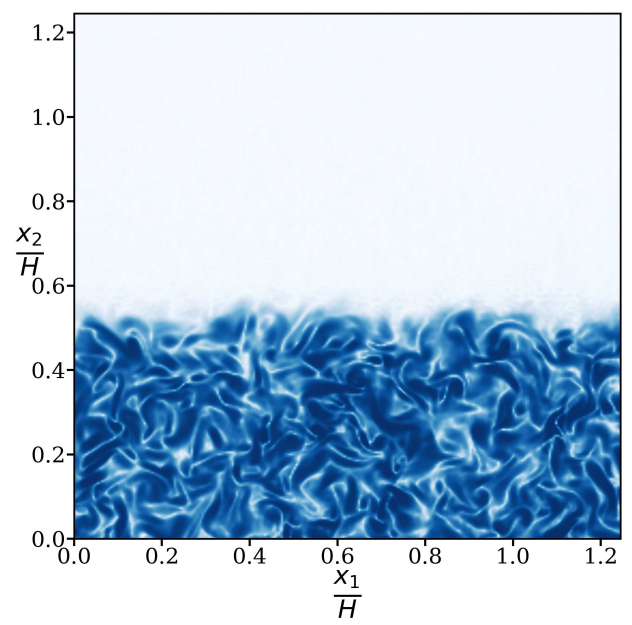

(a) $K 4$ for $\tau=31.43$

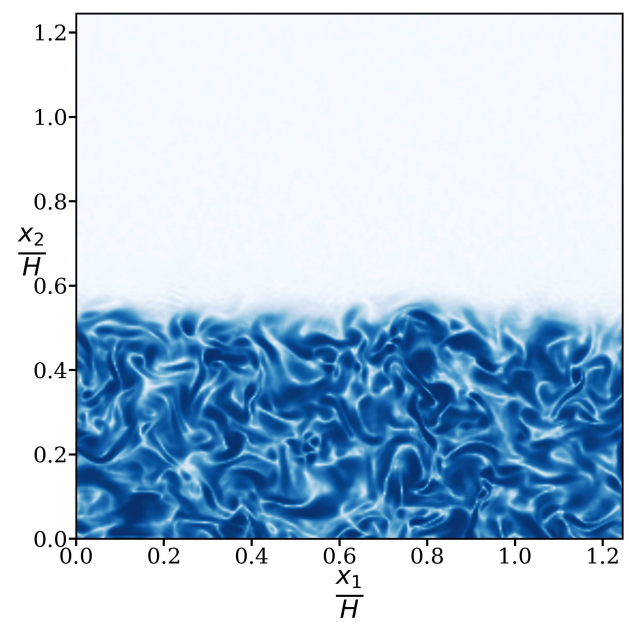

(b) $K 2$ for $\tau=33.78$

Figure 6: Zoom of the enstrophy contours in the $\left(x_{1}, x_{2}\right)$ plane near the TNTI for the $K 2$ and $K 4$ simulations at similar time instants. The figures show only half of the turbulent region $H / 2$.

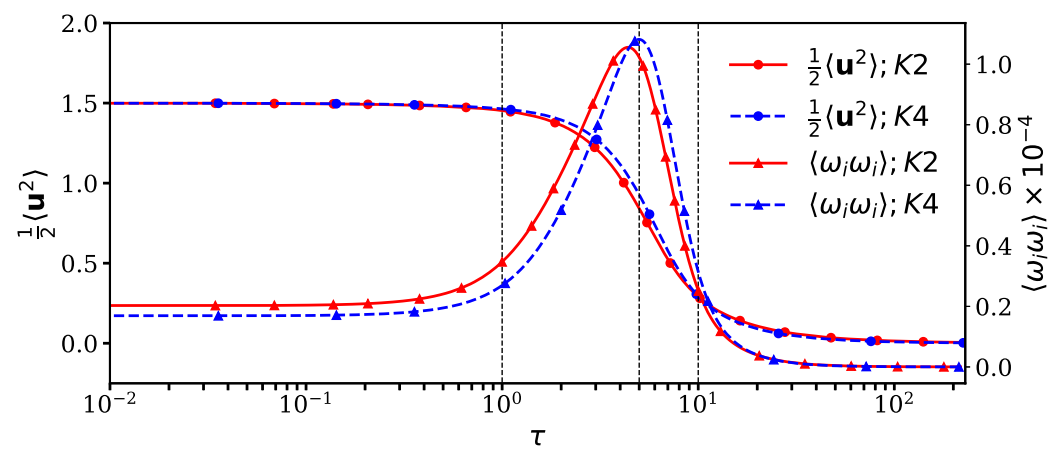

Figure 7: Evolution of turbulent kinetic energy, $\frac{1}{2}\left\langle\mathbf{u}^{2}\right\rangle$, and enstrophy $\left\langle\omega_{i} \omega_{i}\right\rangle$, in the $K 4$ and $K 2$ simulations of DHIT.

$$
\frac{1}{2}\left\langle\mathbf{u}^{2}\right\rangle \sim \tau^{-m}
$$

In practice the exponent $m$ was computed as,

$$
m(\tau)=\frac{\tau}{\frac{1}{2}\left\langle\mathbf{u}^{2}\right\rangle} \frac{\partial \frac{1}{2}\left\langle\mathbf{u}^{2}\right\rangle}{\partial \tau},
$$

where a gaussian filter was applied to the mean kinetic energy signal prior to the computation of the derivative in Eq. (3.3), so as to remove any possible numerical noise in this computation.

In agreement with the theoretical predictions, the turbulent kinetic energy tends to a power law decay with $\frac{1}{2}\left\langle\mathbf{u}^{2}\right\rangle(\tau) \sim \tau^{-m}$, where $m$ approaches the theoretical values of 


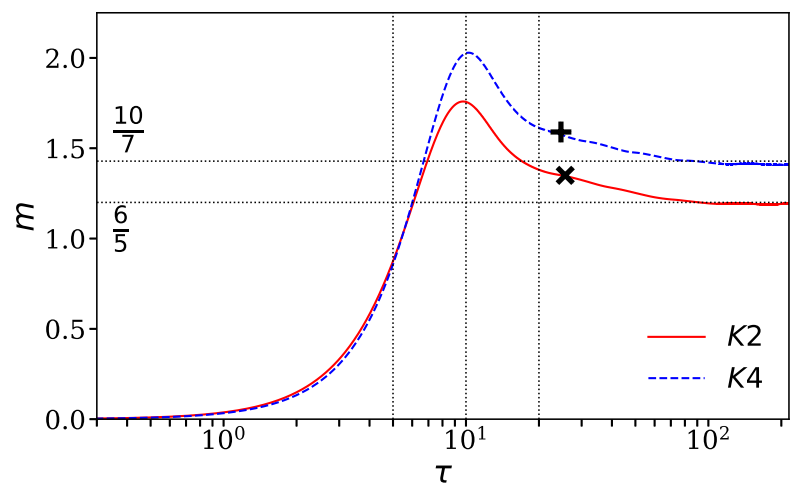

Figure 8: Temporal evolution of the kinetic energy decay exponent $m$ defined in Eq. (3.2) for the simulations $K 4$ and $K 2$. The two crosses represent the initialisation points for the subsequent SFT simulations, $\tau_{S F T}$.

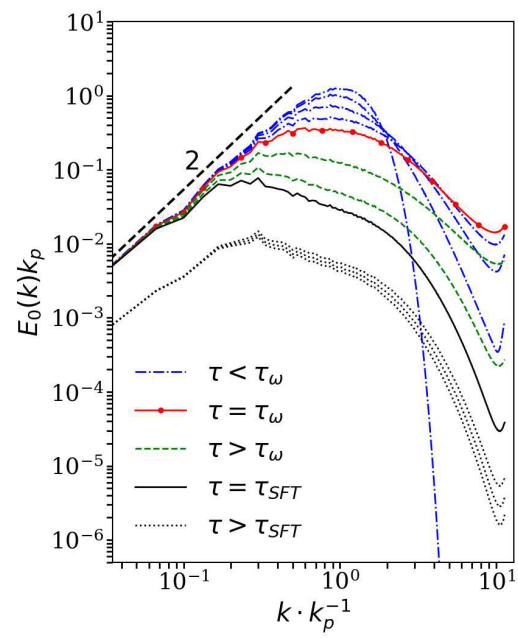

(a) $K 2$

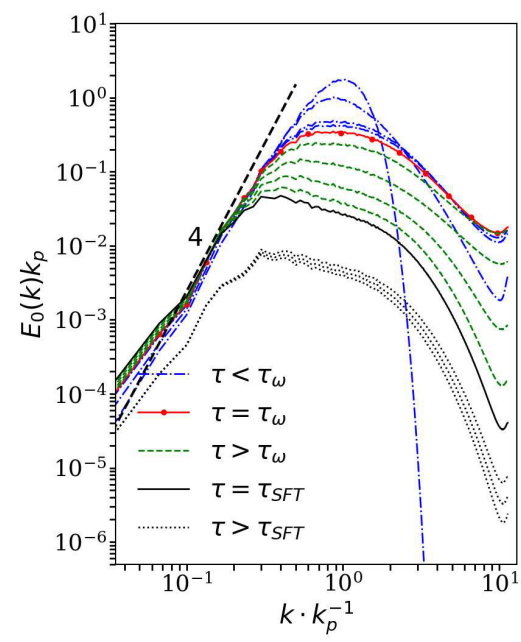

(b) $K 4$

Figure 9: Kinetic energy spectrum for the simulations $K 2$ and $K 4$ at several instants: at the initial stages of the simulation $\left(t<t_{\omega}\right)$, at the enstrophy peak $\left(t=t_{\omega}\right)$, after the enstrophy peak $\left(t>t_{\omega}\right)$, at the time instant used to initialise the SFT simulations $\left(t=t_{S F T}\right)$, and during the SFT simulation $\left(t>t_{S F T}\right)$.

$10 / 7$ and 6/5 for the simulations $K 4$ and $K 2$, respectively (Davidson 2004; Ishida et al. 2006).

Finally, figure 9 shows the kinetic energy spectrum for the $K 4$ and $K 2$ simulations at several instants. These spectra are consistent with similar observations made in many previous DNS of decaying isotropic turbulence (Lesieur 1997). Initially $\left(\tau<\tau_{\omega}\right)$ we observe the typical filling up of the kinetic energy at high wave numbers, for both simulations, until, by the time the peak enstrophy is attained $\left(\tau=\tau_{\omega}\right)$ the wave number range is fully developed. After this $\left(\tau>\tau_{\omega}\right)$ it is clear that the mean kinetic energy is decaying, since the magnitude of the kinetic energy spectra decreases for all wavenumbers 


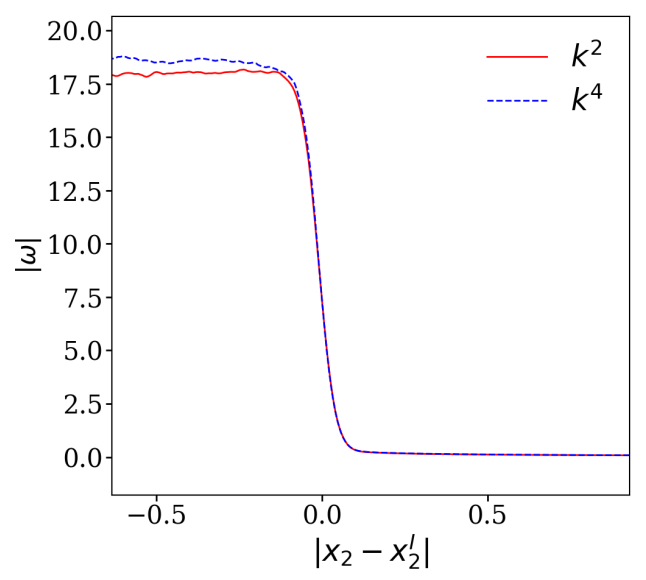

Figure 10: Mean profiles of enstrophy $\left\langle\omega_{i} \omega_{i}\right\rangle$ for the SFT simulations generated from the velocity fields for the $K 2$ and $K 4$ simulations, at $\tau=26.30$ and $\tau=24.69$, respectively. The normal coordinate $\left(x_{2}\right)$ is subtracted by the initial position of the TNTI $\left(x_{2}^{I}\right)$, which separates the flow field into the irrotational $\left(x_{2}-x_{2}^{I}<0\right)$ and turbulent $\left(x_{2}-x_{2}^{I}>0\right)$ flow regions.

and $\frac{1}{2}\left\langle\mathbf{u}^{2}\right\rangle=\int_{0}^{\infty} E(k) d k$. Concomitantly with this, the spectrum peak is seen to be shifting towards smaller wave numbers, for both simulations, while the spectra slope in the infrared region is preserved i.e. $E(k) \sim k^{n}$, where $n$ is constant during the decay, even during the SFT simulation. However (for $\tau \gtrsim 5$ ) while the energy peaks shift towards smaller wave numbers, the extent of the region where the power law $E(k) \sim k^{n}$ is observed decreases. In the case of the $K 2$ simulation this occurs at a faster pace than with the $K 4$ simulation. Since the shape of the kinetic energy spectrum in this region determines the profiles of $\left\langle u_{i} u_{i}\right\rangle$ in the irrotational region of SFT simulations, it is necessary that the initialisation of the SFT simulations be made prior to the extinction of this region. For this reason, the $K 2$ simulation of SFT has to be started before $m$ attains its theoretical value of $m=6 / 5$. On the other hand for the $K 4$ simulation, although the $E(k) \sim k^{n}$ region is conserved during a longer period of time, $R e_{\lambda}$ decreases to significantly low values. Thus, also for this case, the initialisation of the corresponding SFT simulation is started prior to point where theoretical value of $m=10 / 7$ is attained, even though both DHIT simulations are carried out the longest possible time after the enstrophy peak. These considerations determine the time instants from the DHIT simulations that were used to generate the initial conditions for the two SFT simulations, which are $\tau=26.25$ and $\tau=24.65$, for the $K 2$ and $K 4$ simulations, respectively, and which are indicated by crosses in figure 8 . The resolution of the velocity fields at these instants is $k_{\max } \eta \sim 1.2$ for both simulations, where $k_{\max }$ is the maximum wave number.

Figure 10 shows the profiles of mean enstrophy $\left\langle\omega_{i} \omega_{i}\right\rangle$ used in the analysis of SFT, which were generated by the instantaneous fields stemming from the $K 2$ and $K 4$ simulations, as described above. These profiles are computed with a single instantaneous field, where the averaging comes from spatial averages done in the $x_{1}$ and $x_{3}$ homogeneous directions, and moreover, the samples are duplicated by adding the data from the two (upper and lower) TNTIs for each simulation. In these and the following figures the normal coordinate $\left(x_{2}\right)$ is subtracted by the initial position of the TNTI $\left(x_{2}^{I}\right)$, that separates the flow field into the irrotational $\left(x_{2}-x_{2}^{I}<0\right)$ and turbulent $\left(x_{2}-x_{2}^{I}>0\right)$ 


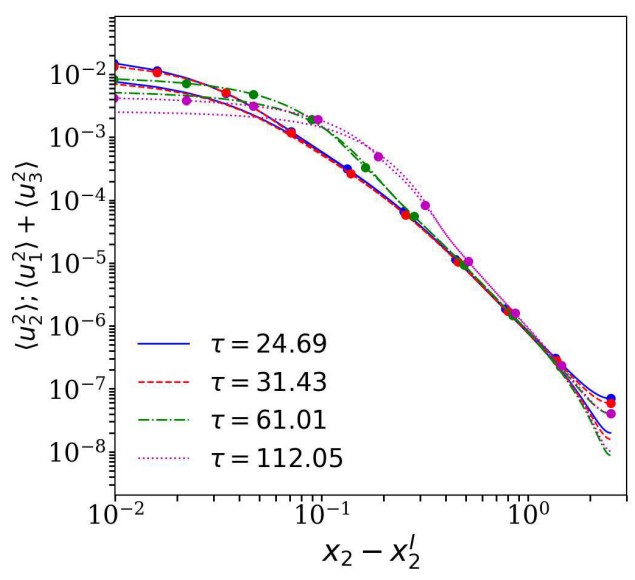

(a) Simulation $K 4$.

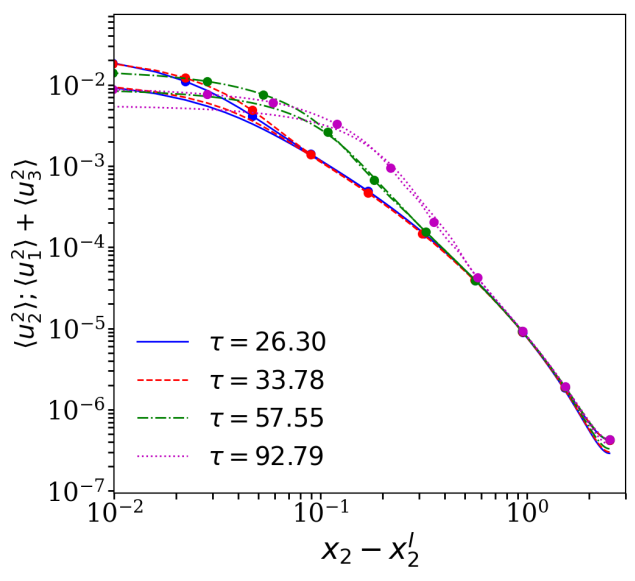

(b) Simulation $K 2$.

Figure 11: Mean profile of variance of the velocity components $\left\langle u_{2}^{2}\right\rangle$ (lines without symbols) and $\left\langle u_{1}^{2}\right\rangle+\left\langle u_{3}^{2}\right\rangle$ (lines with symbols), for the SFT simulations generated from the velocity fields for the $K 2$ and $K 4$ simulations at several time instants.

flow regions. Even though the profiles are made using 'classical' statistics and not the conditional averaging used in e.g. Bisset et al. (2002); Westerweel et al. (2009); da Silva et al. (2014) used in many works dealing with the TNTI, they retain the fast enstrophy rise observed at the TNTI $\left(x_{2}-x_{2}^{I}=0\right)$ bridging the enstrophy from the irrotational and turbulent flow regions.

Figure 11 shows the mean profiles of $\left\langle u_{2}^{2}\right\rangle$ and of $\left\langle u_{1}^{2}\right\rangle+\left\langle u_{3}^{2}\right\rangle$, from the SFT simulations, at several times. The results show that for sufficiently large distances from the TNTI layer $\left(\left(x_{2}-x_{2}^{I}\right) / L>>1\right)$ the relation $\left\langle u_{2}^{2}\left(x_{2}\right)\right\rangle=\left\langle u_{1}^{2}\left(x_{2}\right)\right\rangle+\left\langle u_{3}^{2}\left(x_{2}\right)\right\rangle$ predicted by Phillips (1955), and its independence from the shape of the kinetic energy spectrum in the infrared region, does hold in the present SFT simulations. A slight deviation from this relation only occurs for very large distances from the TNTI where the effects of the periodic boundary conditions start to influence the statistics.

This completes the assessment of the DNS of DHIT and SFT. The next section will focus on the verification of the new theoretical relations derived in the present work.

\section{Assessment of the new theoretical results in DNS of SFT}

We now turn to the verification of the new theoretical results using the $K 2$ and $K 4$ DNS from the SFT phase of the simulations.

Figure 12 shows mean profiles of variance of the normal velocity $\left\langle u_{2}^{2}\left(x_{2}\right)\right\rangle$ for several time instants, obtained from the two SFT simulations used in the present work, which were generated from the DHIT simulations initialised with energy spectra with $E(k) \sim k^{2}$ and $E(k) \sim k^{4}$ (in the infrared region). At the instants chosen for the analysis $(\tau \approx$ $25-60$ ) the turbulent Reynolds number in both simulations is $R e_{\lambda} \approx 30$, which is also close to the value used in similar analyses of decaying isotropic turbulence (Ishida et al. 2006; Teixeira \& da Silva 2012). More importantly, as we have shown above the present theoretical results are independent of the magnitude of the Reynolds number and therefore the present results are generally valid and of general interest to higher Reynolds number flows. 


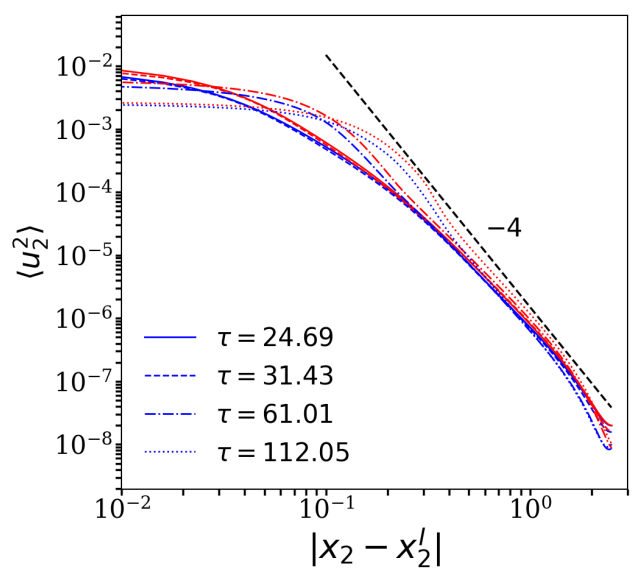

(a) $K 4$ for several time instants.

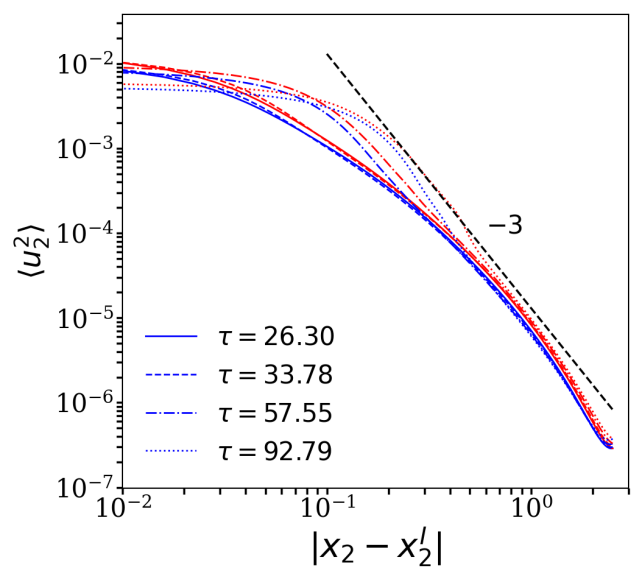

(b) $K 2$ for several time instants.

Figure 12: Profiles of normal velocity variance $\left\langle u_{2}^{2}\left(x_{2}\right)\right\rangle$, in the NT region for the SFT simulations $K 2$ and $K 4$ at several time instants, computed for the lower $\left(x_{2}<0\right.$ red) and upper $\left(x_{2}>0\right.$ - blue) regions. The profiles exhibit regions with power laws of $\left\langle u_{2}^{2}\left(x_{2}\right)\right\rangle \sim x_{2}^{-4}$ and $\left\langle u_{2}^{2}\left(x_{2}\right)\right\rangle \sim x_{2}^{-3}$ for the simulations with $n=4$ and $n=2$, respectively.

Clearly, the form of the kinetic energy in the infrared region determines the decay of the velocity fluctuations in the non-turbulent region, as expected from the discussion in section 2. Specifically, the mean profiles confirm that in the non-turbulent region the normal velocity variance decays as $\left\langle u_{2}^{2}\left(x_{2}\right)\right\rangle \sim x_{2}^{-4}$ and $\left\langle u_{2}^{2}\left(x_{2}\right)\right\rangle \sim x_{2}^{-3}$, for initial energy spectra with $E(k) \sim k^{4}$ and $E(k) \sim k^{2}$, respectively, in the infrared region. Similar results are observed for the other velocity components (not shown).

Both profiles exhibit power law regions for large distances from the TNTI, and cease to obey this power law region when approaching the periodic boundary conditions imposed at the edges of the computational domain. Indeed, the small upturn observed at the end of the profiles is a non-physical phenomenon that arises from the periodic boundary conditions required in the present DNS using pseudo-spectral schemes, however these conditions do not prevent us from observing the power law regions predicted by the new theoretical results, as shown here.

Figure 13 shows similar profiles for the Taylor micro-scale in the normal direction $\lambda_{2}$, which is expected to have the same asymptotic behaviour to the Taylor micro-scale defined in section 2 (Teixeira \& da Silva 2012). The advance of the TNTI is clearer here than in the previous figure, and shows that for both simulations ( $K 2$ and $K 4)$ and for the three times, the mean profile of $\lambda_{2}$ exhibits a power law region with $\lambda_{2} \sim x_{2}$ in agreement with the results discussed in section 2 .

Finally, figure 14 shows the profiles for the viscous dissipation rate $\varepsilon$. Again the advance of the TNTI is clearly observed in both cases, and again the figures show that for both simulations and for the three times, the mean profile of $\varepsilon$ exhibits a power law region with $\varepsilon \sim x_{2}^{-6}$ and $\varepsilon \sim x_{2}^{-5}$, for the simulations with a kinetic energy spectrum with $E(k) \sim k^{4}$ and $E(k) \sim k^{2}$, respectively, again in agreement with the results discussed in section 2 .

It is noteworthy that the asymptotic behaviour for the Taylor micro-scale and viscous dissipation predicted by Phillips (1955) for the more usual case of $E(k) \sim k^{4}$ is observed 


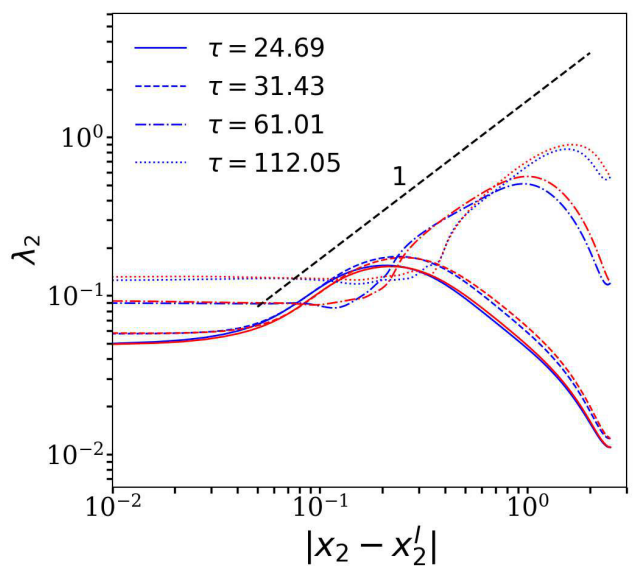

(a) $K 4$ for several time instants.

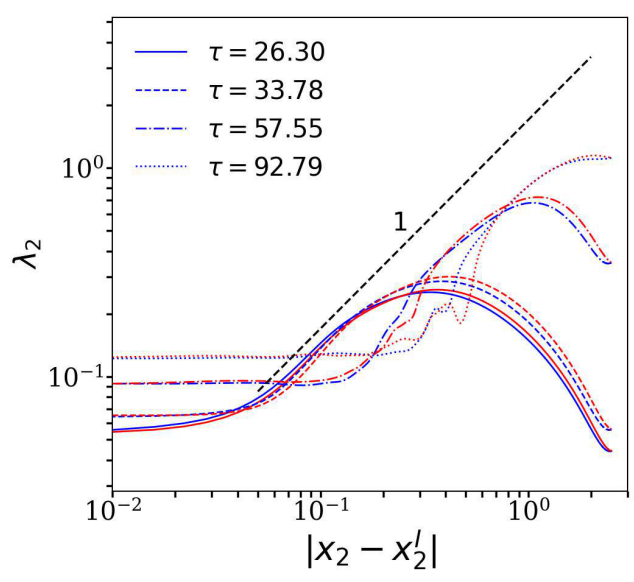

(b) $K 2$ for several time instants.

Figure 13: Profiles of Taylor micro-scale $\lambda_{2}$, in the NT region for the SFT simulations $K 2$ and $K 4$ at several time instants, computed for the lower $\left(x_{2}<0-\right.$ red) and upper $\left(x_{2}>0\right.$ - blue) regions. The profiles exhibit regions with power laws of $\lambda_{2} \sim x_{2}$ for both simulations (with $n=4$ and $n=2$ ).

in the present data, but has been very rarely reported before in either experimental data or numerical simulations, and therefore attests the robustness of the present DNS, and at the same time that the behaviour of these variables for a spectrum with $E(k) \sim k^{2}$ is also recovered.

The results from this section confirm that the theoretical results derived more than half a century ago by Phillips (1955) constitute in fact a particular case of a more general decay law.

\section{Conclusions}

More than half a century ago Phillips (1955) derived several scaling laws associated with the irrotational motions arising in the non-turbulent region near a turbulent/nonturbulent interface (TNTI) bounding a turbulent flow, such as a turbulent jet, turbulent wake, or a turbulent boundary layer. Defining as $x_{2}$ the distance from the TNTI, Phillips (1955) deduced that sufficiently far away from the interface, typically for distances bigger than $x_{2}>L$ where $L$ is the integral scale of turbulence from the nearby turbulent region, the kinetic energy of the potential velocity fluctuations decays as $\left\langle u_{i}^{2}\right\rangle \sim x_{2}^{-4}$, with $i=1,2,3$, while the tangential $u_{1}$, and $u_{3}$, and normal $u_{2}$, velocity fluctuations in relation to the TNTI, obey the law $\left\langle u_{2}^{2}\left(x_{2}\right)\right\rangle=\left\langle u_{1}^{2}\left(x_{2}\right)\right\rangle+\left\langle u_{3}^{2}\left(x_{2}\right)\right\rangle$.

In the present work it is shown that this result is in fact a particular case of a more general law whereby the potential velocity fluctuations decay as $\left\langle u_{i}^{2}\right\rangle \sim x_{2}^{-m}$, for $i=1,2,3$, where $m$ is a power law related to the kinetic energy spectrum at low wave numbers, $E(k) \sim k^{n}$. Specifically, if the turbulent flow region bordering the TNTI displays a Batchelor $\left(E(k) \sim k^{4}\right)$ or Saffman $\left(E(k) \sim k^{2}\right)$ spectrum, the decay of the velocity fluctuations will satisfy $\left\langle u_{i}^{2}\right\rangle \sim x_{2}^{-4}$, and $\left\langle u_{i}^{2}\right\rangle \sim x_{2}^{-3},(i=1,2,3)$ respectively. Moreover, the relation $\left\langle u_{2}^{2}\left(x_{2}\right)\right\rangle=\left\langle u_{1}^{2}\left(x_{2}\right)\right\rangle+\left\langle u_{3}^{2}\left(x_{2}\right)\right\rangle$ holds independently of the shape of the spectrum in the infrared region. The new decay laws have been confirmed in DNS of shear-free turbulence i.e. turbulent flows where a TNTI layer separates a turbulent 


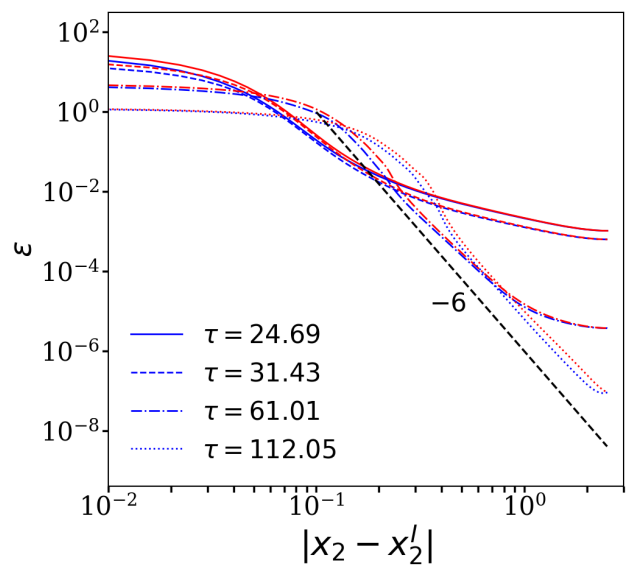

(a) $K 4$ for several time instants.

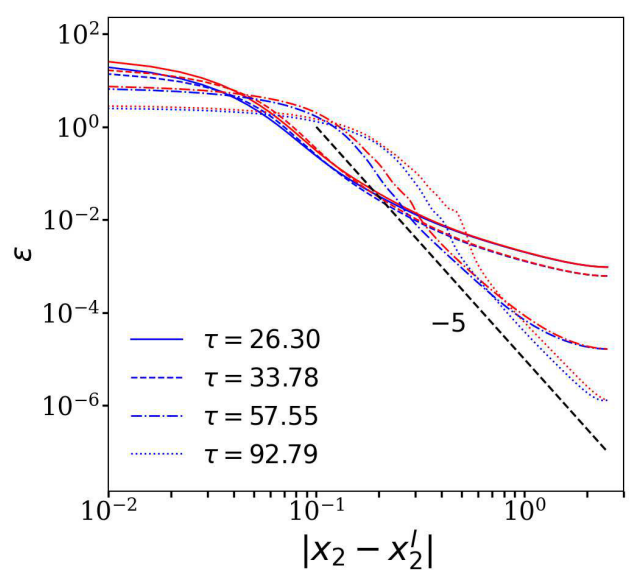

(b) $K 2$ for several time instants.

Figure 14: Profiles of viscous dissipation rate $\varepsilon$, in the NT region for the SFT simulations with $K 2$ and $K 4$ at several time instants, computed for the lower $\left(x_{2}<0-\right.$ red) and upper $\left(x_{2}>0\right.$ - blue) regions. The profiles exhibit regions with power laws of $\varepsilon \sim x_{2}^{-6}$ and $\varepsilon \sim x_{2}^{-5}$ for the simulations with $n=4$ and $n=2$, respectively.

region from a region of non-turbulent (irrotational) motion, evolving in the absence of mean shear. The new theoretical results extend not only the asymptotic relations from Phillips (1955) and Carruthers \& Hunt (1986), but also the ones from Teixeira \& da Silva (2012). Specifically, it was demonstrated that the profile of mean viscous dissipation rate follows $\varepsilon \sim x_{2}^{-6}$ and $\varepsilon \sim x_{2}^{-5}$ for a Batchelor and a Saffman spectrum, respectively, while the Taylor micro-scale $\lambda_{i} \sim x_{2}$ (for both cases), the latter not being dependent on the particular form of the kinetic energy spectrum in the infrared region.

Finally, it is confirmed that the asymptotic behaviour of the velocity fluctuations in the non-turbulent region depends only on the infrared region of the kinetic energy spectrum. Specifically, the new asymptotic results are robust and insensitive to particular changes in the form of the kinetic energy spectrum for wave numbers $k>k_{p}$, where $k_{p}$ is the energy spectrum peak, as well as the value of $k_{p}$ itself and the extent of the wave number region $k>k_{p}$, which indirectly reflects also the lack of sensitivity of these results to the magnitude of the Reynolds number.

Arguably, the asymptotic results should be valid also for TNTIs arising from other flow types, and including mean shear, such as turbulent planar jets or wakes, provided the kinetic energy spectra in the infra-red wave number range exhibits a Batchelor or a Saffman spectrum.

\section{Declaration of interests}

The authors report no conflict of interest.

\section{Acknowledgements}

C. B. da Silva acknowledges Fundação para a Ciência e Tecnologia (FCT) through IDMEC, under LAETA, project UIDB/50022/2020. The authors acknowledge Minho Advanced Computing Center for providing HPC computing and consulting 
resources that have contributed to the research results reported within this paper (https://macc.fccn.pt). The authors acknowledge the Laboratory for Advanced Computing at University of Coimbra for providing HPC, computing and consulting resources (http://www.lca.uc.pt).

\section{REFERENCES}

Antonia, R. A., Shah, D. A. \& Browne, L. W. B. 1987 The organized motion outside a turbulent wake. The Physics of Fluids 30 (7), 2040-2045.

Batchelor, G. K. 1953 The Theory of Homogeneous Turbulence. Cambridge University Press.

Birkhoff, G. 1954 Fourier synthesis of homogeneous turbulence. Communications on Pure and Applied Mathematics 7 (1), 19-44.

Bisset, D. K., Hunt, J. C. R. \& Rogers, M. M. 2002 The turbulent/non-turbulent interface bounding a far wake. J. Fluid Mech. 451, 383-410.

Bradbury, L. J. S. 1965 The structure of a self-preserving turbulent plane jet. Journal of Fluid Mechanics 23 (1), 31-64.

Bradshaw, P. 1967 Irrotational fluctuations near a turbulent boundary layer. Journal of Fluid Mechanics 27 (2), 209-230.

Canuto, C., Hussaini, M. Y., Quarteroni, A. \& Zang, T. A. 1987 Spectral Methods in Fluid Dynamics. Springer-Verlag.

Carruthers, D. J. \& Hunt, J. C. R. 1986 Velocity fluctuations near an interface between a turbulent region and a stably stratified layer. J. Fluid Mech. 165, 475-501.

Cimarelli, A., Cocconi, G., Frohnapfel, B. \& Angelis, E. De 2015 Spectral enstrophy budget in a shear-less flow with turbulent/non-turbulent interface. Phys. Fluids 27, 125106.

Davidson, P. A. 2004 Turbulence, an introduction for scientists and engineers. Oxford University Press.

Eyink, G. L. \& Thomson, D. J. 2000 Free decay of turbulence and breakdown of self-similarity. Phys. Fluids 12, 477-479.

FABRIS, G. 1979 Conditional sampling study of the turbulent wake of a cylinder. part 1. J. Fluid Mech. 94 (4), 673-709.

IshidA, T., DAvidson, P. A. \& Kaneda, Y. 2006 On the decay of isotropic turbulence. J. Fluid Mech. 564, 455-475.

Kovasznay, L. S. G., Kibens, V. \& Blackwelder, R. F. 1970 Large-scale motion in the intermittent region of a turbulent boundary layer. J. Fluid Mech. 41 (2), 283-325.

Lesieur, M. 1997 Turbulence in fluids, third ed.. Kluwer.

Oberlack, M. \& Zieleniewicz, A. 2013 Statistical symmetries and its impact on new decay modes and integral invariants of decaying turbulence. Journal of Turbulence 14 (2), 4-22.

Perot, B. \& Moin, P. 1995 Shear-free turbulent boundary layers. part 1. physical insights into near-wall turbulence. J. Fluid Mech. 295, 199-227.

Phillips, O. M. 1955 The irrotational motion outside a free turbulent boundary. Proc. Camb. Phil. Soc. 51, 220.

Pope, S. B. 2000 Turbulent Flows. Cambridge University Press.

Saffman, P.G. 1967 The large-scale structure of homogeneous turbulence. J. Fluid Mech. 27, 581-593.

Da Silva, C. B., Hunt, J.C.R., Eames, I. \& Westerweel, J. 2014 Interfacial layers between regions of different turbulent intensity. Annu. Rev. Fluid Mech. 46, 567-590.

Da Silva, C. B., dos Reis, R. J. N. \& Pereira, J. C. F. 2011 The intense vorticity structures near the turbulent/non-turbulent interface a jet. J. Fluid Mech. 685, 165-190.

Silva, T. S. \& DA Silva, C. B. 2017 The behaviour of the scalar gradient across the turbulent/non-turbulent interface in jets. Phys. Fluids 29, 085106.

Silva, T. S., Zecchetto, M. \& DA Silva, C. B. 2018 The scaling of the turbulent/nonturbulent interface at high reynolds numbers. J. Fluid Mech. 843, 156-179.

Sunyach, M. \& Mathieu, J. 1969 Zone de melange d'un jet plan fluctuations induites dans le cone a potentiel-intermittence. International Journal of Heat and Mass Transfer 12 (12), $1679-1697$. 
TAveiRA, R. R. \& DA Silva, C. B. 2013 Kinetic energy budgets near the turbulent/nonturbulent interface in jets. Phys. Fluids 25, 015114.

Teixeira, M.A.C. \& DA Silva, C. B. 2012 Turbulence dynamics near a turbulent/nonturbulent interface. J. Fluid Mech. 695, 257-287.

Thomas, R. M. 1973 Conditional sampling and other measurements in a plane turbulent wake. J. Fluid Mech. 57 (3), 549-582.

VAssilicos, J.C. 2011 An infinity of possible invariants for decaying homogeneous turbulence. Physics Letters A 375 (6), 1010 - 1013.

Watanabe, T., Jaulino, R., Taveira, R., da Silva, C.B., Nagata, K. \& Sakai, Y. 2017 Role of an isolated eddy near the turbulent/non-turbulent interface layer. Physical Review Fluids 2.

Watanabe, T., Sakai, Y., Nagata, K., Ito, Y. \& Hayase, T. 2014 Enstrophy and passive scalar transport near the turbulent/non-turbulent interface in a turbulent planar jet flow. Phys. Fluids 26, 105103.

Westerweel, J., Fukushima, C., Pedersen, J. M. \& Hunt, J. C. R. 2005 Mechanics of the turbulent-nonturbulent interface of a jet. Physics Review Letter 95, - .

Westerweel, J., Funushima, C., Pedersen, J. M. \& Hunt, J. C. R. 2009 Momentum and scalar transport at the turbulent/non-turbulent interface of a jet. J. Fluid Mechanics, Cambridge University Press 631, 199-230.

Williamson, J. H. 1980 Low-storage runge-kutta schemes. J. Comp. Phys. 35, 48-56.

Wygnanski, I. \& Fiedler, H. E. 1970 The two-dimensional mixing region. J. Fluid Mech. $41(2), 327-361$. 